\title{
Measurements of luminosity and normalised beam-induced background using the CMS Fast Beam Condition Monitor
}

\author{
Nathaniel Odell*i \\ On behalf of the CMS Collaboration \\ Northwestern University \\ Evanston, IL 60208-3112 USA \\ E-mail: Nathaniel.Jay. Odell@cern.ch
}

\begin{abstract}
The CMS Beam Conditions and Radiation Monitoring system (BRM) is installed to protect the CMS detector from high beam losses and to provide feedback to the LHC and CMS on the beam conditions. The Fast Beam Condition Monitor (BCM1F), one of the sub-detectors in the BRM system, is installed inside the pixel volume close to the beam pipe and consists of two planes of 4 modules each located $1.8 \mathrm{~m}$ away from the IP, on both ends. It uses single-crystal CVD diamond sensors, radiation hard front-end electronics and an optical transmission of the signal. It is designed for single particle rate measurements, detecting both machine induced beam background and collision products on a bunch-by-bunch basis.

Presented is the implementation of the normalised online beam-induced background measurement and the online instantaneous luminosity measurement. The method for determining the luminosity from the measured rates, including the absolute calibration using the Van der Meer scan, and the measurement performance will be described.
\end{abstract}

36th International Conference on High Energy Physics,

July 4-11, 2012

Melbourne, Australia

\footnotetext{
* Speaker.

${ }^{\dagger}$ Thanks to the members of the CMS Beam and Radiation Group whose guidance and hard work made this possible. Particular thanks to Anne Dabrowski, David Stickland, and Olga Novgorodova.
} 


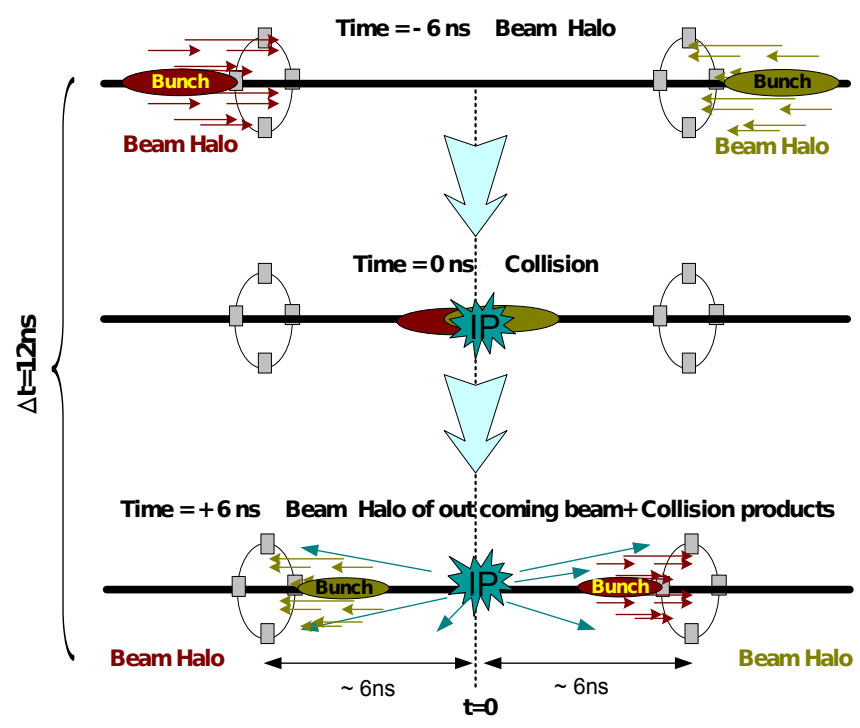

Figure 1: Schematic of arrival time for various beam products.

\section{Introduction}

The CMS experiment [1] must function in an exceptionally high radiation environment with an expected maximum instantaneous luminosity in excess of $10^{34} \mathrm{~cm}^{-2} \mathrm{~s}^{-1}$, and beam intensities on the order of $10^{14}$ protons. In the interest of protecting the inner detector - in particular, the inner silicon pixel tracker - it is important to characterize the long-term dose, and respond to rapid beam losses. To meet these needs, several beam and radiation detectors have been placed along the beam-pipe near the CMS detector.

Of particular interest is the Fast Beam Conditions Monitor (BCM1F). This detector is highly responsive to rapid variations in the beam conditions with a time resolution on the order of several nanoseconds. In addition to providing a vital role in beam monitoring, the detector is also capable of providing online, bunch-by-bunch instantaneous luminosity which can be used to provide realtime feedback to the LHC.

\section{Beam Conditions Monitoring at CMS}

The Fast Beam Conditions Monitor, BCM1F [2], is a particle detector based on single-crystal diamonds grown using chemical vapour (CVD) deposition techniques.

Some important properties of single-crystal diamonds (sCVD) are its radiation hardness due to its large displacement energy and low leakage current with almost no temperature dependence removing the need for active cooling. These qualities make it an attractive material to be used in a particle detector, especially in areas of high radiation and locations where space is limited. This is particularly relevant in the densely packed CMS forward region, or in the CMS inner region where access to services like cooling is inconvenient. In addition, diamond sensors are characterised by very fast response allowing temporal measurements with nanosecond resolution. 


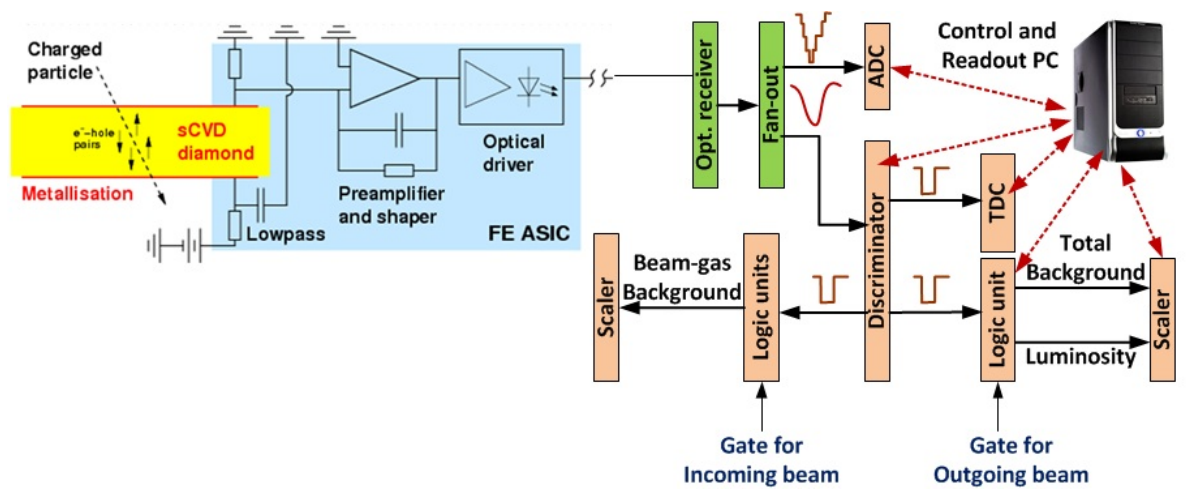

Figure 2: A schematic of the BCM1F readout chain

The BCM1F detector consists of $8 \mathrm{sCVD}$ sensors, installed in two planes located at $1.8 \mathrm{~m}$ on either end of the interaction point (IP) with each module at transverse distance of $5 \mathrm{~cm}$ from the nominal beam spot . Each sensor has a surface area of $5 \times 5 \mathrm{~mm}$ and a thickness $0.5 \mathrm{~mm}^{3}$.

The distance between the sensors and the IP is optimal for the separation of incoming and outgoing particles and corresponds to a time-of-flight of $\sim 6 \mathrm{~ns}$ for relativistic particles as can be seen in fig. 1. Hence, by dividing the signals into different time windows the rates of beam halo particles can be seperated from those of collision products.

The details of the BCM1F readout chain are described in detail in [3]. In summary, an analog optical signal is sent from a radiation hard front-end ASIC to our back-end electronics (shown in fig. 2) where rate counting and data acquisition for offline analysis of pulse characteristics is done.

\section{BCM1F Signal Characterization}

Several studies were undertaken over the course of data-gathering at CMS to characterize the behaviour of BCM1F. The goal of these tests is to characterize the detector's sensitivity to a variety of beam products and stability over a range of different operating conditions. Given that the BCM1F sends an analog signal to the backend, pulse characteristics (i.e., pulse amplitudes and time to return to threshold) can be assessed offline by using an ADC VME module with nanosecond resolution. It is important that this is understood since the method for discriminating, and therefore, counting rates, uses a fixed-threshold discriminator. This means that if there occurs a shift in the signal baseline we are not going to see signals since they will no longer show sufficient voltage to pass the pre-set threshold. One way to gauge the significance of this effect is to measure the time that a given pulse spends over the threshold.

An example the characterization is shown in fig. 3. One important feature to note is the occasional high amplitude signal that results in saturation of the front-end electronics. The consequence of this is a decrease in the hit counting efficiency due to an increase the time required to recover to baseline.

Another important quantity to determine is the single bunch hit probability. This is done by counting the rate of hits in the lead bunch in an orbit. This bunch is preceded by the abort gap, and is therefore much less likely to be affected by inefficiencies caused by signals from preceding bunches. A fair amount of variation between each of the 8 sensors is observed. Given that a range 


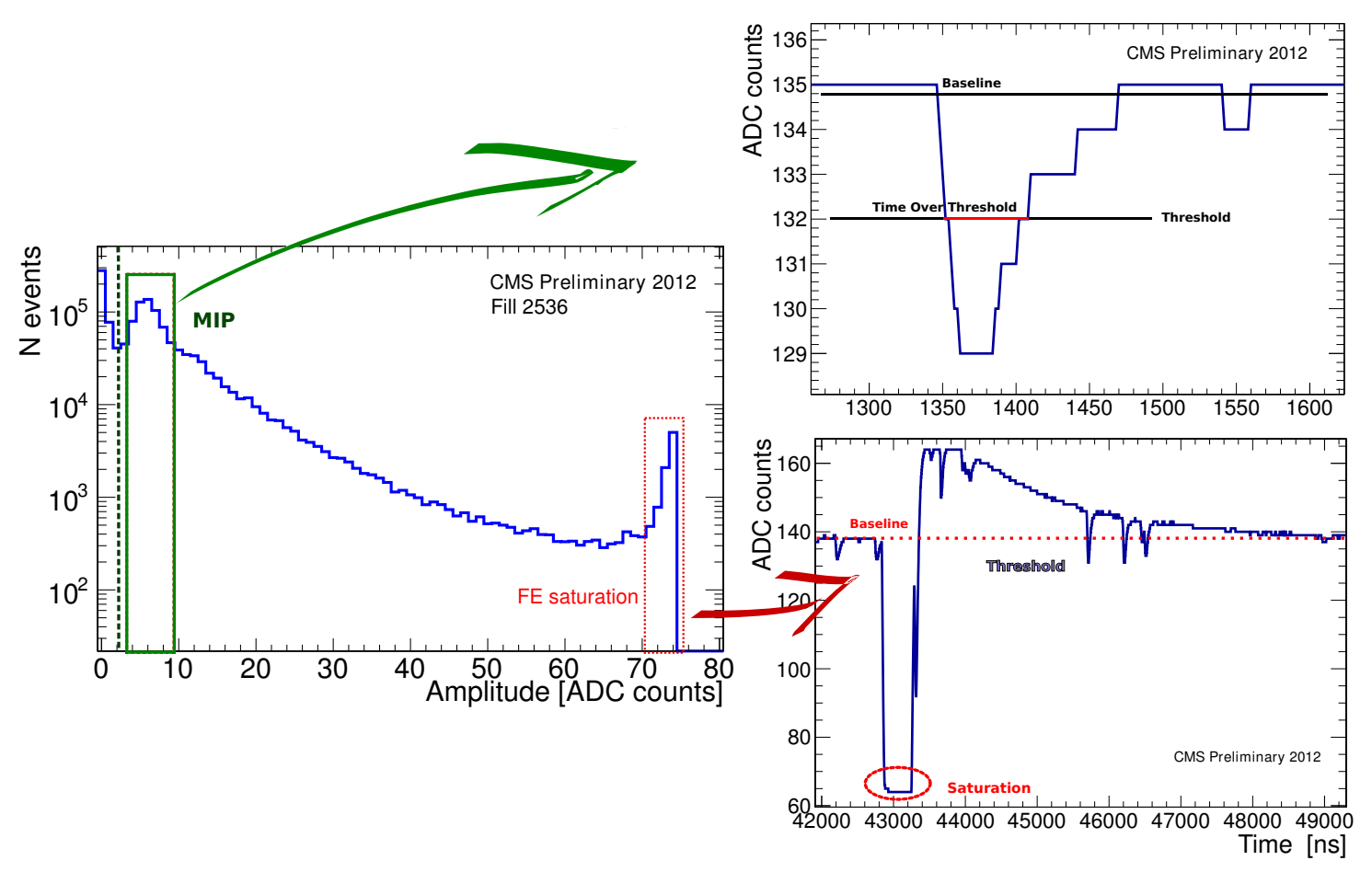

Figure 3: Pulse characterization from data collected with back-end ADC.

of single diamond rates need be considered in order to cover the range of observed luminosities, it is important to accurately characterize how the relative differences in hit probabilities depends on the particle flux. For the most part, it is observed that they are stable over the range of observed luminosities and demonstrate a linear dependence (fig. 4).

\section{Beam-induced background measurement}

So-called beam-gas interactions occur when protons from the beam interact with residual gas in the beam pipe. These interactions are dominated by small angle, inelastic collisions with the resulting particles moving parallel to the beam pipe. Typically the flux of these type of interactions is on the order of $10^{-5}$ less than the flux from collision products. In the case that there is degradation of the vacuum in the beam pipe, this flux can increase significantly causing excessive firing of channels in the pixel detector resulting in an increase in the overall dead-time.

Because the flux relative to collision products is typically so low, beam-gas products are separated from collision products by only looking at non-colliding bunch crossings that precede colliding bunches. Additionally, only non-colliding bunches that are sufficiently separated from the previous bunch train are considered due to the high flux from albedo following colliding bunches crossing.

An example of the resulting measurement is shown in fig. 5. The normalized beam gas flux measurement is implemented online independent of the CMS central data acquisition, by using the following steps 


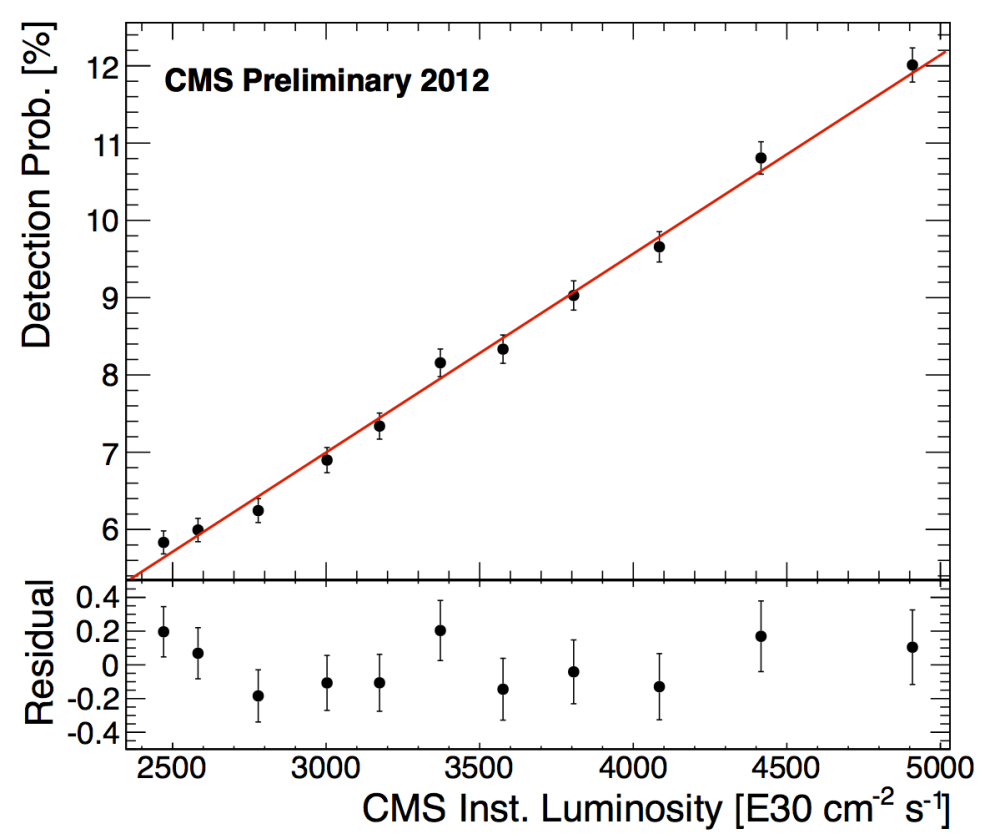

Figure 4: Single bunch hit probabilities as a function of the instantaneous luminosity measured by HF. Hit probability is measured for the leading bunch in each orbit.

- In real-time measure the bunch pattern of beam 1 and beam 2 with the BPTX.

- Select non-colliding bunches by generating an $8 \mathrm{~ns}$ wide gate from the BPTX signal, coincident with the time arrival of incoming non-colliding bunches in BCM1F.

- Open a VETO gate, $900 \mathrm{~ns}$ long after a colliding bunch, thus permitting only non-colliding bunch intervals, well separated from collisions (reduce albedo products) to be selected.

- Use $12 \mathrm{~ns}$ time of flight between the $-\mathrm{Z}$ and $+\mathrm{Z}$ BCM1F detector planes to gate separately on incoming and outgoing beam products.

- Measure a pedestal rate in $40 \mathrm{~ns}$ window before non-colliding bunch train, to estimate the non-beam gascomponent of the measured rate due to activation from collision products.

- Measure the bunch charge for each selected non-colliding bunch, online using the Fast Beam Current Transformer (FBCT), so as to obtain a correctly normalized beam-gas flux measurement, per 1011 particles.

The NIM logic implemented for the beam gas measurement is shown schematically in fig. 5 . The count rates in the relevant gates are integrated with a scaler module and then published every second. The normalized beam-gas flux is calculated, and made available to both CMS and the LHC every second.

The performance of the beam-gas measurement can be seen for LHC fill 2104 in fig. 5. For additional details on this measurement, see [4]. 

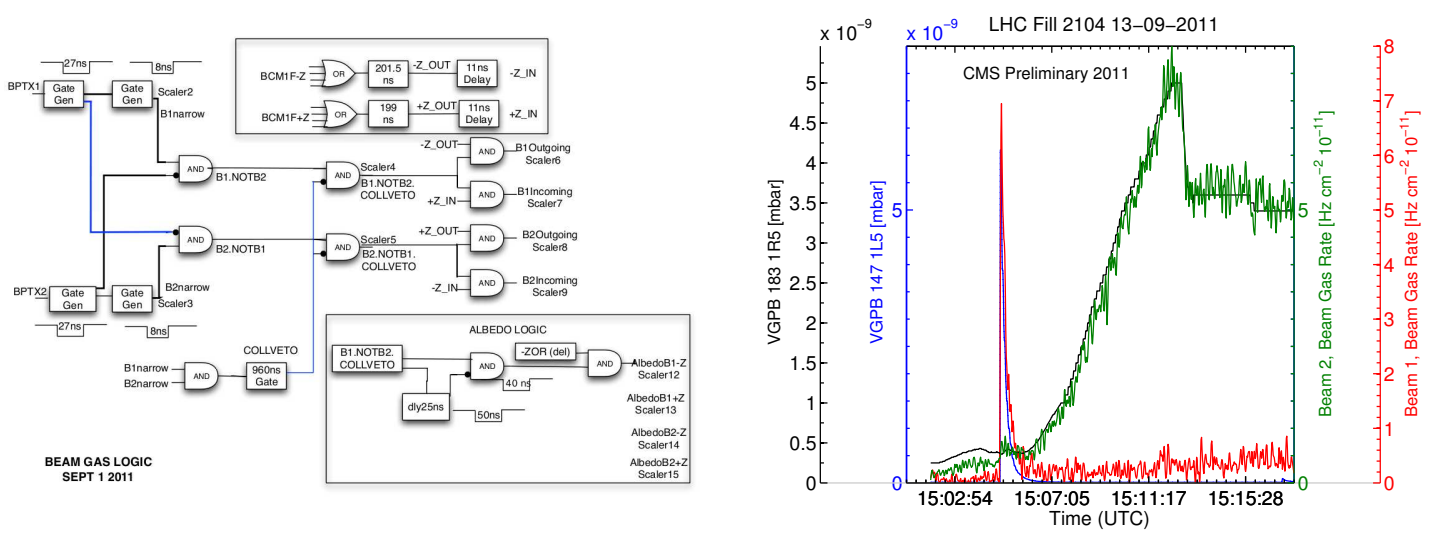

Figure 5: Schematic of BCM1F back-end gating to select on beam-gas products (left). Comparison of beam-gas measurement and vacuum quality using pressure gauges (right).

\section{Luminosity Determination and Calibration}

The method of determining the luminosity delivered to CMS relies upon a relation between measurable quantities (rates in the BCM1F detector, for instance) and the actual rate of interactions due to collisions inside the detector. In the ideal circumstance with $n_{b}$ colliding bunches circulating in the accelerator the following relation can be used,

$$
L=\frac{n_{b} f_{b} \mu}{\sigma_{\text {inel }}}
$$

where $f_{b}$ is the frequency of the orbit, $\sigma_{\text {inelastic }}$ is the inelastic scattering cross-section, and $\mu$ is the average number of interactions per bunch crossing,

$$
\mu=\frac{\langle N\rangle}{n_{b}}
$$

This, of course, assumes that the average number of interactions per orbit $(\langle N\rangle)$ is perfectly measured. In reality, a quantity $\mu_{o b s}$ is measured and is related to $\mu$ by multiplication by some detection efficiency, $\varepsilon$, such that,

$$
\begin{aligned}
\mu_{o b s} & =\varepsilon \mu, \\
\sigma_{o b s} & =\varepsilon \sigma_{\text {inelastic }} .
\end{aligned}
$$

The $\varepsilon$ term accounts for all detector specific effects, which may depend on the luminosity. Even at constant per bunch luminosity, $\varepsilon$ is a function of the number of bunches in LHC (arises from recovery time of front end: with more bunches in the machine, hits from a given bunch are more likely to be screened by a preceding bunch and are more likely to screen following bunches), the bunch intensity and the pattern of bunches within an orbit. Therefore, the relation between luminosity and the measured quantities is written,

$$
L=\frac{n_{b} f_{b} \mu_{o b s}}{\sigma_{o b s}} .
$$


The following sections describe our methodology for determining $\mu_{o b s}$ and $\sigma_{o b s}$.

\subsection{Zero Counting}

The number of interactions per bunch crossing for a given set of beam parameters follows a Poisson distribution. The probability of a given number of interactions, $n$, is given by,

$$
p(n)=\frac{\mu^{n} e^{-\mu}}{n !}
$$

This relation allows to determine the average number of interactions when the probability that there is no interaction, $p(0)$, is measured,

$$
\begin{aligned}
\mu & =-\ln [p(0)] \\
& =-\ln [1-p(>0)]
\end{aligned}
$$

For the case of the $\mathrm{BCM} 1 \mathrm{~F}, p(0)$ is defined as the probability that there are no hits in the eight diamond sensors.

\subsection{Van der Meer Scan Calibration}

In order to convert the measured $\mu_{o b s}$ value to a luminosity, we need to determine an absolute luminosity calibration. This, in essence, consists of determining the $\sigma_{o b s}$ value. The approach that we have adopted for producing the calibration was proposed by Van der Meer [5] and relies on making a simultaneous measurement of $\mu_{o b s}$ and horizontal and vertical beam separation. The following expression defines the luminosity purely in terms of beam parameters,

$$
L=f_{b} N_{1} N_{2} \int \rho_{1}(x, y) \rho_{2}(x, y) \mathrm{d} x \mathrm{~d} y
$$

where $N_{1 / 2}$ are the particles in the bunches of the beams, and $\rho_{1 / 2}(x, y)$ the density of the beams as a function of the transverse distance from their respective centers. Assuming that there is no correlation between the beam density in the $x$ and $y$ directions, the above equation can be reparameterized in terms of the effective beam width,

$$
\begin{aligned}
& \Sigma_{x}=\left[2 \pi \int \rho_{1}(x) \rho_{2}(x) \mathrm{d} x\right]^{-1}, \\
& \Sigma_{y}=\left[2 \pi \int \rho_{1}(y) \rho_{2}(y) \mathrm{d} y\right]^{-1},
\end{aligned}
$$

yielding,

$$
L=\frac{f_{b} N_{1} N_{2}}{2 \pi \Sigma_{x} \Sigma_{y}}
$$

From this expression and eq. 5.5, a relation which allows for the determination of the effective cross-section can be derived, 

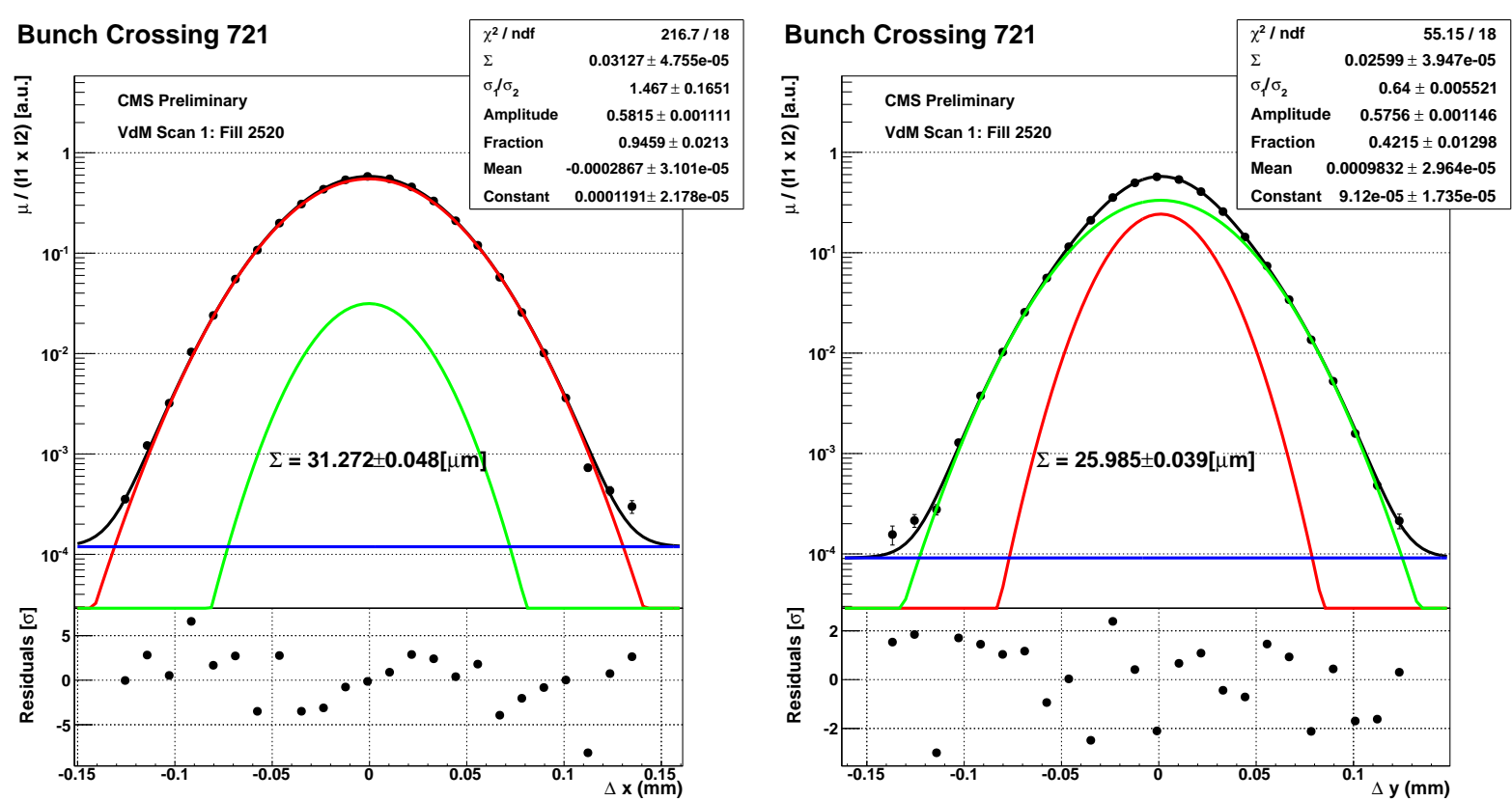

Figure 6: Data gathered during the April 2012 VdM scan. The data is fit with a double gaussian plus constant where the single gaussian components are shown in red and green, the constant in blue, and the combined result is in black.

$$
\sigma_{v i s}=2 \pi \Sigma_{x} \Sigma_{y} \frac{\mu_{v i s, \max }}{N_{1} N_{2}} .
$$

The effective widths are determined by separating the beams independently in the $\mathrm{x}$ and $\mathrm{y}$ directions. Therefore, for each calibration two separate scans need to be carried out. As the beams are separated, the rate at the detector is measured giving us a relation between the value of $\mu$ and the beam separation. This is then fit to extract the relevant quantities. For the April 2012 scan, it was determined that a double Gaussian distribution gives a suitably good fit. The form of the fit functions is,

$$
\mu_{x, v i s}=\mu_{x, v i s}^{\text {peak }}\left(\frac{f_{1} \Sigma_{x}}{\sigma_{1}} e^{\frac{\left(x-X_{0}\right)^{2}}{2 \sigma_{1}^{2}}}+\left(1-\frac{f_{1} \Sigma_{x}}{\sigma_{1}}\right) e^{\frac{\left(x-X_{0}\right)^{2}}{2 \Sigma_{x}^{2}}\left(1-f_{1}\right)^{2}\left(1-\frac{f_{1} \Sigma_{x}}{\sigma_{1}}\right)^{2}}\right)
$$

The BCM1F participated in the April 2012 VdM scan that consisted of 3 separate scans of the beam in the $x$ and $y$ directions. An example of the extraction of the calibration constant is shown in 6. In figure 7, the results of the first scan for each bunch crossing is shown with a comparison to the other luminometers from CMS. A systematically smaller width than HF is observed, but this difference is at the level of $1 \%$. Though the cause of this is not completely understood, a possible explanation lies in the higher sensitivity of HF to lower rates.

\section{Simulation}

To model and eventually correct for observed inefficiencies in the bunch-by-bunch counting rates a MC simulation was developed. This simulation takes into account pulse characteristics 

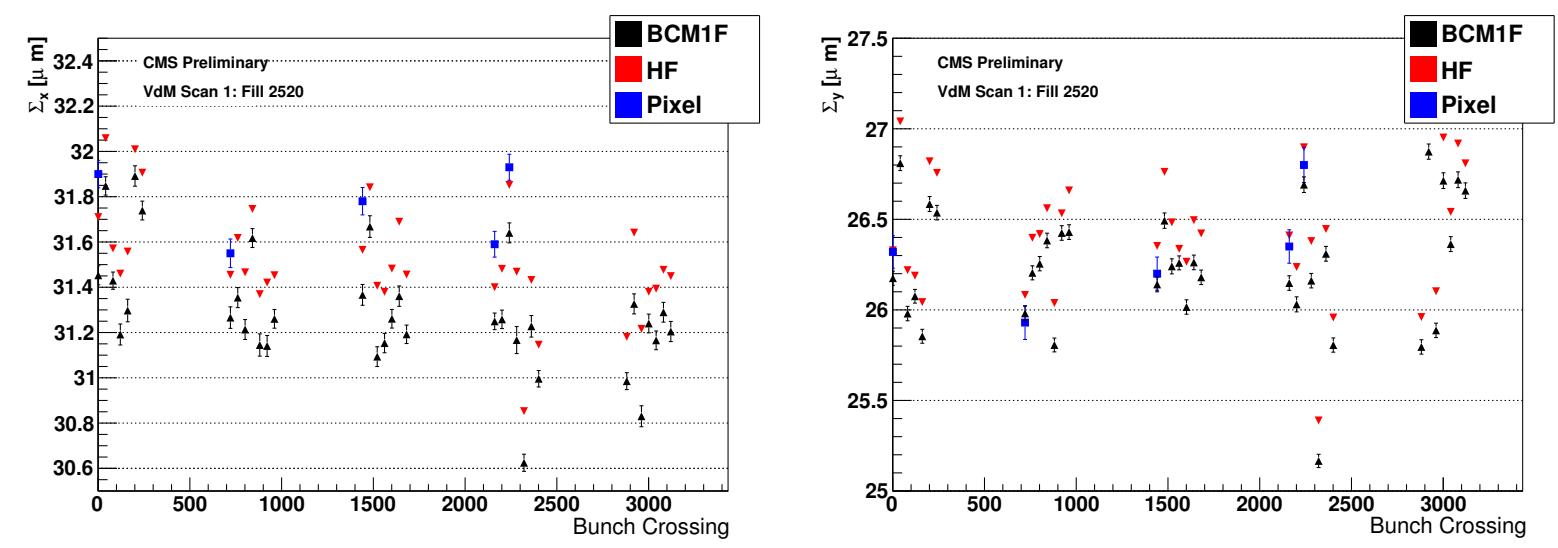

Figure 7: Comparison between CMS luminometers of width of beam overlap for $x$ and $y$ scans.
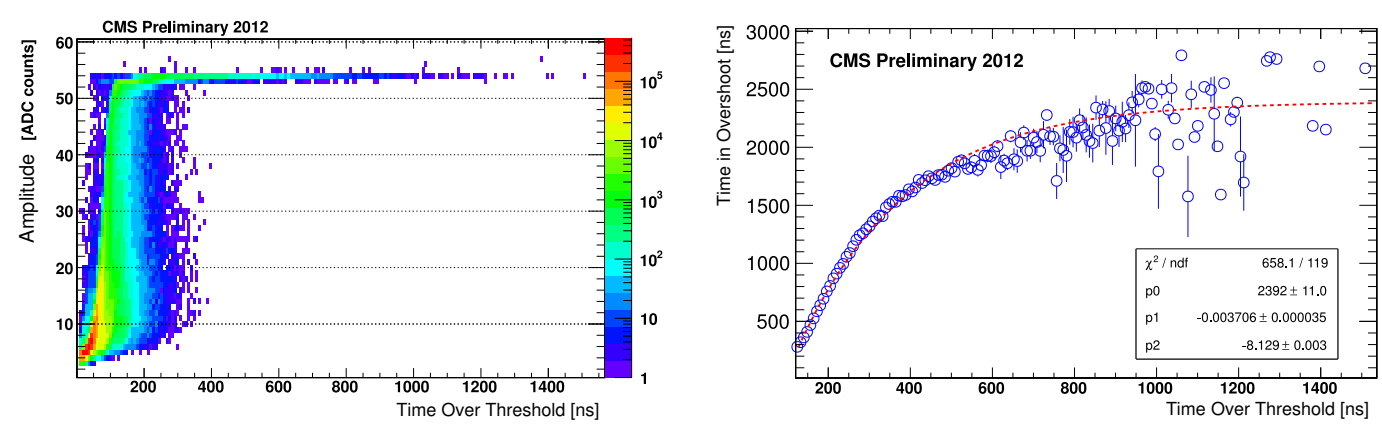

Figure 8: Examples of distributions that are generated from the ADC analysis and used as inputs to the detector simulation.

extracted from the ADC-based analysis. Input parameters for the simulation are:

- diamond-by-diamond single bunch hit probability

- time over threshold

- correlation between time over threshold/pulse amplitude and time in overshoot

- evolution of overshoot amplitude

Examples of the distributions that were used as inputs are shown in fig. 8. The simulation is based on the hypothesis that the main source of inefficiency when counting bunch-by-bunch or integrated rates is that hits in bunches that come earlier in the fill pattern tend to "screen" hits from later bunches since the front-end electronics has not recovered from the previous hit. This is exacerbated by very energetic deposits that cause front-end saturation and lead to very long recovery times. In figure 9 a comparison of the model and data collected while running with 1380 filled bunches is shown for the lead 400 bunch crossings. As can be seen, the overall drop off as a function of bunch crossing (roughly exponential) is reproduced in the simulation quite well. There is quite a bit of bunch-by-bunch variation that is not reproduced in the simulation as modelling these effects is outside the purview of this study. 


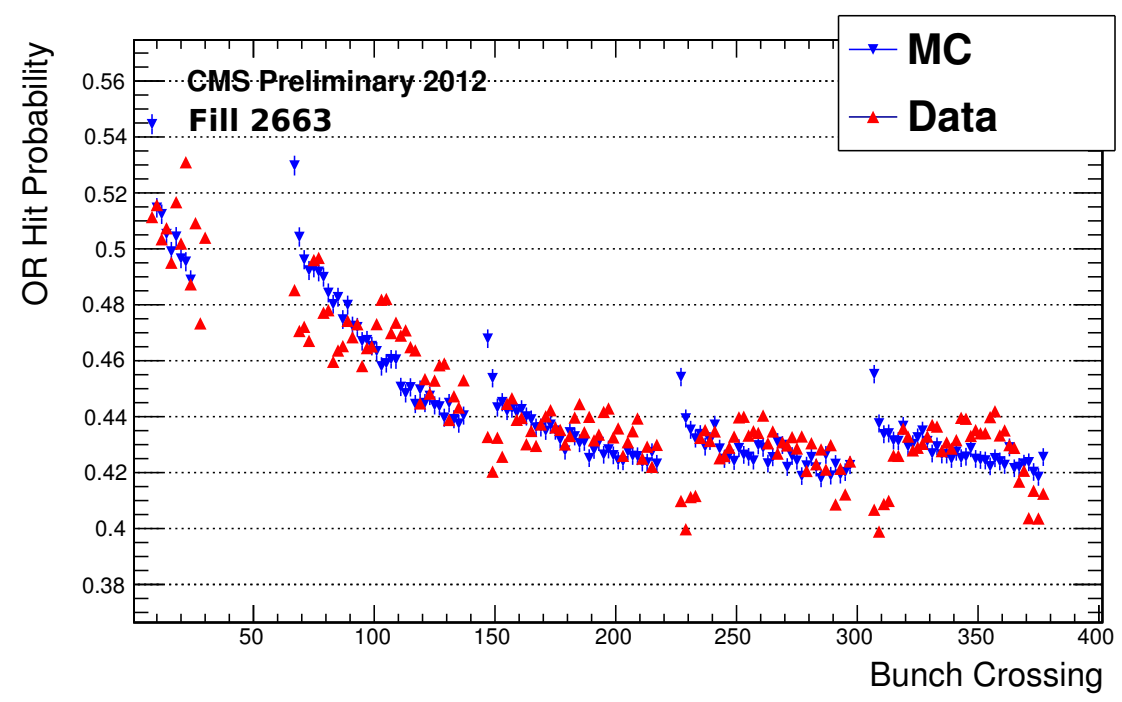

Figure 9: Results of simulation of the BCM1F counting rate as a function of bunch-crossing for fill pattern with 1380 filled bunches, 1331 of which are colliding at IP5.

\section{Conclusions}

The BCM1F has demonstrated its effectiveness in measuring rates of both primary and secondary beam products during the first two years of running, and continues to do so into the third year of LHC running. It has proven valuable as a beam background, and has shown its viability as a luminometer.

Several studies are under way to assess radiation damage, the dependance of gain on rate, and effects of bias voltage polarization. The result of the knowledge gathered so far will guide plans for upgrades during the long shut-down of 2013-2014, and allow us to improve performance during the remainder of 2012. Plans for the upcoming shut-down include the installation of at least 8 new diamond sensors with the primary improvements being made on the construction of the front-end electronics. Some of the planned features of the new readout chip are,

- peaking time $\leq 10 \mathrm{~ns}$

- $\mathrm{FWHM} \leq 10 \mathrm{~ns}$

- Baseline recovery $\leq 30 \mathrm{~ns}$

These improvements will make the BCM1F more robust in general and increase its viability as the primary online luminosity monitor at CMS. 


\section{References}

[1] The CMS Collaboration, The CMS experiment at the LHC, Journal of Instrumentation 3 (2008) S08004 doi:10.1088/1748-0221/3/08/S08004

[2] R. Hall-Wilton et al., Fast beam conditions monitor (BCM1F) for CMS, 2008 IEEE Nuclear Science Symposium Conference Record, pp. 3298-3301.

[3] A. Bell et al., Fast Beam Conditions Monitor BCM1F for the CMS Experiment, Published in Nucl.Instrum.Meth. A614 (2010) 433-438 DESY-09-178. Nov 2009. 16 pp. e-Print: arXiv:0911.2480 [physics.ins-det]

[4] A. Dabrowski et al, The Performance of the Beam Conditions and Radiation Monitoring System of CMS, proceedings submitted at IEEE Nuclear Science Symposium, Oct. 2011, Valencia Spain; CMS note: CMS CR-2011/275

[5] Van der Meer, S., Calibration of the effective beam height in the ISR, CERN-ISR-PO/68-31(1968).

[6] E. Castro et al, The CMS Beam Conditions and Radiation Monitoring System, proceedings of the TIPP 2011 conference in Chicago, 2011, submitted.

[7] R. Walsh, Performance of the CMS Fast Beam Conditions Monitoring, proceedings of the 2010 IEEE Nuclear Science Symposium Conference.

[8] J. Kaplon and W. Dabrowski, Fast CMOS binary front end for silicon strip detectors at LHC experiments, 2005 IEEE Transactions on Nuclear Science 52(6), pp.2713-2720. 